\title{
CARACTERIZAÇÃO DAS ATIVIDADES DE ENFERMAGEM EM SAÚDE COLETIVA DESENVOLVIDAS NA REGIONAL NORTE DE SAÚDE DO DISTRITO FEDERAL
}

\author{
PUBLIC HEALTH NURSING PRACTICES DEVELOPED IN THE NORTHERN \\ REGIONAL HEALTH UNIT OF THE FEDERAL DISTRICT \\ CARACTERIZACIÓN DE LAS ACTIVIDADES DE ENFERMERIA EN SALUD \\ COLECTIVA DESARROLLADAS EN LA REGIONAL NORTE DE SALUD \\ DEL DISTRITO FEDERAL
}

Helena Eri Shimizu ${ }^{1}$

Erlita Rodrigues dos Santos ${ }^{2}$

RESUMO:O estudo teve como objetivo identificar e analisar as açōes desenvolvidas pela equipe de enfermagem em saúde coletiva na Regional Norte de Saúde do Distrito Federal. Para a coleta de dados aplicou-se um questionário com a descrição das diversas atividades cotidianas da assistência a 29 enfermeiras, 91 auxiliares de enfermagem e 42 agentes de saúde. Para a melhor compreensão de como as atividades são desenvolvidas realizou-se grupos focais com cada uma das categorias profissionais. Os resultados mostram que todos elementos da equipe empregam grandes esforços para organizar, controlar e atender a demanda espontânea e ainda executar as atividades de auxilio às ações médicas, consequentemente priorizam o atendimento individual em detrimento do coletivo.

PALAVRAS-CHAVE: práticas de enfermagem, Saúde Coletiva, Enfermagem

\section{INTRODUÇÃO}

O conhecimento do que a equipe de enfermagem desenvolve nos serviços de saúde sempre foi uma preocupação da Enfermagem brasileira. Ao examinar os estudos voltados para essa temática, deparamo-nos com um número razoavelmente grande de trabalhos que analisam as atividades desenvolvidas pelas enfermeiras, e também com alguns estudos mais voltados para as atividades realizadas pelos demais elementos da equipe de enfermagem.

Os estudos que identificam o fazer das enfermeiras concluem que estas têm desenvolvido, em maior proporção, as atividades administrativas em detrimento das assistenciais. Tal constatação foi analisada no Seminário Nacional sobre Currículo do Curso de Graduação de Enfermagem, realizado em 1986, como "desvio" de função da enfermeira, pois a mesma deveria estar realizando mais "funções assistenciais que seriam as de enfermagem propriamente ditas e que independem da autoridade de outro profissional e que fornecem um caráter de especificidade à profissão" (SEMINÁRIO NACIONAL SOBRE CURRICULO DO CURSO DE GRADUAÇĀO DE ENFERMAGEM, 1986).

Alguns estudos recentes, que analisam as práticas das enfermeiras especificamente na área de saúde coletiva, confirmam que estas continuam desenvolvendo com maior freqüência

${ }^{\dagger}$ Enfermeira. Doutora em Enfermagem. Pesquisadora do Projeto CIPESC. Profa. Adjunta do Departamento de Enfermagem da Faculdade de Ciências da Saúde da Universidade de Brasilia.

${ }^{2}$ Enfermeira. Mestre em Educação. Profa. Pesquisadora do Projeto CIPESC. Coordenadora do Curso de Enfermagem da Faculdade JK. Taguatinga -DF 
as atividades gerenciais e administrativas (BERTOLOZZI et al., 1999, MISHIMA et al. 1999, SHIMIZU; SILVA; 1999). Para Bertolozzi et al. (1999), isso ocorre porque o modelo assistencial adotado na unidade de saúde determina, em parte, as práticas de saúde e, consequentemente, as de enfermagem.

Almeida et al. (1997) destacam que, para o alcançar a saúde coletiva nos dias de hoje, "o trabalho de enfermagem tem se diversificado, indo desde o "cuidar" de enfermagem, seja do individuo, da familia e grupos da comunidade, passando pelas ações educativas e administrativas e ainda a participação no planejamento em saúde" (p.62).

Em 1997, a Associação Brasileira de Enfermagem (ABEn) assumiu o Projeto de Classificação Internacional das Práticas em Saúde Coletiva (CIPESC), tendo como um dos objetivos conhecer as atividades desenvolvidas pela equipe de enfermagem brasileira na área de Saúde Coletiva. Diversas regiões do país compuseram o cenário deste estudo. No Distrito Federal, elegeu-se como Cenário, por atender os critérios do referido projeto, a Regional Norte de Saúde do Distrito Federal que compreende as áreas do Cruzeiro, Asa Norte, Lago Norte, Varjão e Paranoá.

Devido a importância da análise do fazer da enfermagem para melhorar a assistência em saúde coletiva este estudo teve por objetivo:

- Identificar e analisar as atividades desenvolvidas pela equipe de enfermagem (enfermeiros, auxiliares e agentes de saúde) em saúde coletiva, da Regional Norte do Distrito Federal.

\section{METODOLOGIA}

Trata-se de um estudo descritivo e exploratório. A coleta de dados foi realizada por meio de questionário elaborado pelo projeto CIPESC, que continha a descrição de 105 ações, em forma de check-list, desenvolvidas coletiva pelos trabalhadores de enfermagem no cotidiano da assistência em saúde (ANTUNES; SILVA; EGRY et al., 1997). Participaram do estudo 29 enfermeiras, 91 auxiliares de enfermagem e 42 agentes de saúde que trabalham na Regional Norte de Saúde.

Para melhor compreensão de como as atividades são desenvolvidas pelos trabalhadores de enfermagem, foram realizados grupos focais com cada uma das categorias profissionais das equipes dos Centros de Saúde e do Programa Saúde em Casa (PSC), nos quais pedia-se aos sujeitos que explicassem como desenvolviam as atividades por eles descritas no cotidiano dos Serviços de Saúde Coletiva.

As sessões de grupos focais foram gravadas com a autorização dos sujeitos e, posteriormente, transcritas na integra. Para a análise desses dados recorremos a técnica de análise de conteúdo.

\section{APRESENTAÇÃO E DISCUSSÃO DOS RESULTADOS}

Para a apresentação dos dados, optou-se por identificar e analisar as vinte atividades realizadas com maior freqüência e as raramente desenvolvidas pela equipe de enfermagem, pois permitem conhecer o que esses trabalhadores têm realizado, bem como as lacunas existentes nos serviços de saúde coletiva.

No gráfico 1, identifica-se as atividades de organização da infra-estrutura e planejamento do serviço (triagem de pacientes, checagem e reposição de materiais, planejamento e coordenação dos serviços de saúde, organização do fluxo de paciente, controle do pessoal de saúde, supervisão do serviço de limpeza, supervisão da sala de vacina, controle de infecção do ambiente e orientação do público) como as desenvolvidas com maior freqüência pelas enfermeiras. Nos grupos focais as enfermeiras confirmaram que dedicam grande parte do tempo realizando essas atividades. Por um lado, a execução dessas atividades confere satisfação à essas profissionais, pois sentem que têm competência para organizar o serviço, por outro, 
geram desgaste intenso e impedem que executem atividades assistenciais consideradas por elas como as mais relevantes.

\section{GRÁFICO 1 - FREQÜÊNCIA DAS ATIVIDADES DESENVOLVIDAS DIARIAMENTE PELAS EN- FERMEIRAS NO COTIDIANO DOS SERVIÇOS DE SAÚDE COLETIVA}

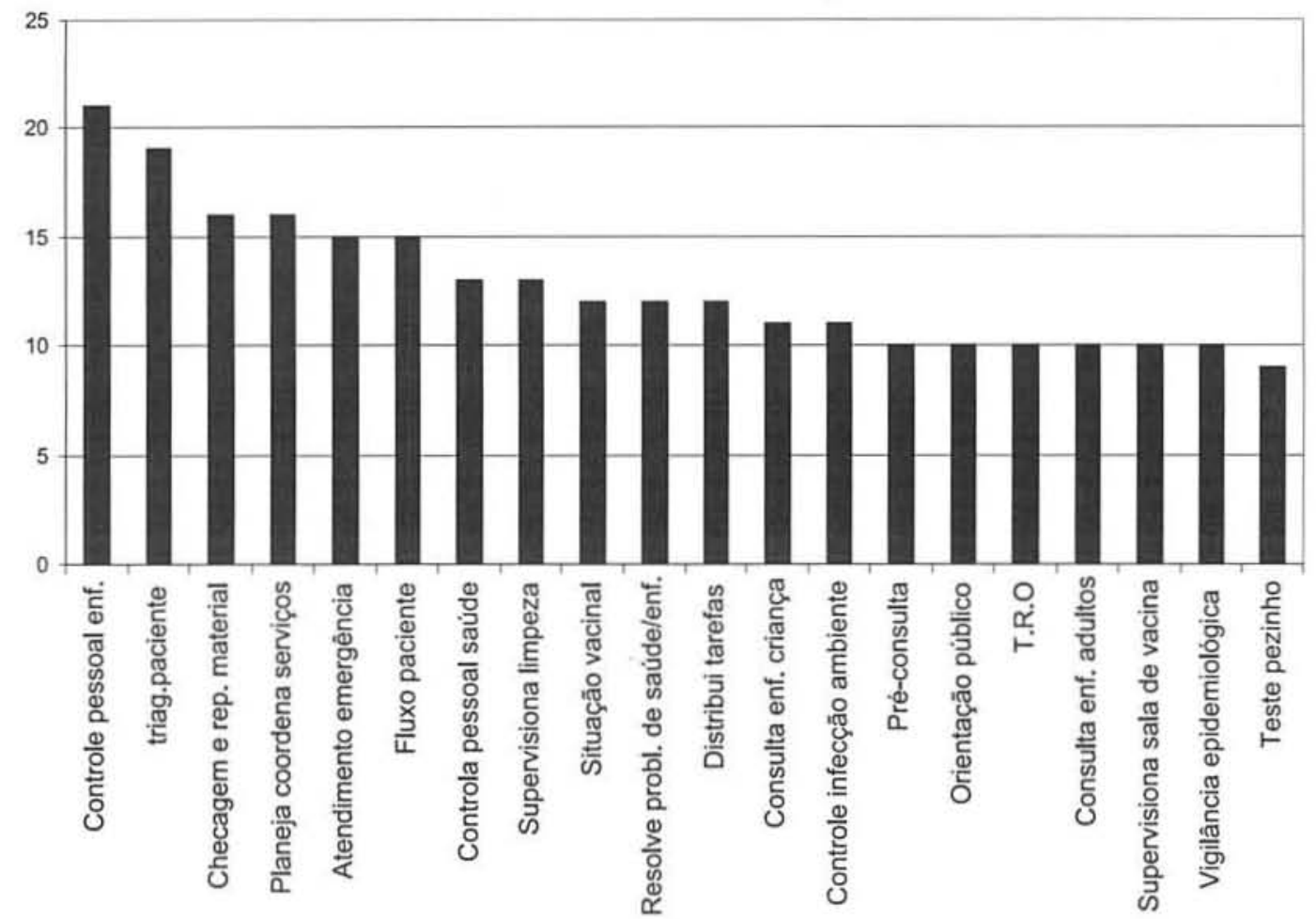

Identificamos também que as enfermeiras desenvolvem, diariamente, algumas atividades de organização e planejamento do trabalho da equipe de enfermagem, a saber: controle de pessoal de enfermagem e distribuição de tarefas. Esses dados mostram que as enfermeiras enfocam a função supervisão no controle das atividades desenvolvidas pelos elementos da equipe de enfermagem.

Das atividades assistenciais especificas da enfermeira desenvolvidas diariamente pelas enfermeiras identificamos a consulta de enfermagem à criança e ao paciente adulto.

Foi possivel perceber, nas sessōes de grupos focais, que as enfermeiras valorizam a consulta de enfermagem. Elas entendem que a consulta permite resolver o problema do paciente, o que Ihes garante autonomia profissional. Entretanto relataram também que a consulta de enfermagem é realizada à pacientes com quadro clínico pouco complexo e, com uma certa freqüência, para "cobrir a ausência do médico". Fica evidente que a consulta de enfermagem tem sido um instrumento de trabalho estrategicamente colocado pelos serviços de saúde do Distrito Federal com a finalidade de aliviar a demanda espontânea para consultas médicas.

Para Araújo (1991), que analisou a resolutividade da consulta de enfermagem, embora esta seja muito valorizada pelas enfermeiras, tem baixo desempenho. Assim, para esta autora não se caracteriza como um instrumento que causa impacto na saúde da população.

Nesse sentido concordamos com Ferreira (1997) que propõe a consulta de enfermagem como um instrumento de trabalho para complementar, no plano individual, outras ações coletivas prioritárias desenvolvidas nos serviços de saúde.

Das atividades assistenciais de caráter complementar ao trabalho do médico, 
identificamos: atendimento de emergência e urgência, TRO, identificação, análise e propostas de soluções para os problemas de saúde e de enfermagem, pré-consulta e teste do pézinho. Evidencia-se que as enfermeiras assumem grande número de atividades assistenciais de maior complexidade e de caráter complementar ao trabalho do médico. Nos grupos focais, as enfermeiras relataram também que redigem a requisição de exames laboratoriais e prescrição de medicamentos, posteriormente assinados pelos médicos.

Por fim, das atividades voltadas para à saúde coletiva, identificamos apenas a avaliação da situação vacinal e a vigilância epidemiológica. Quanto à responsabilidade da enfermeira com o setor de vacinação, Almeida et al. (1997) esclarecem que esta sempre tomou o setor de vacinação como mais de sua responsabilidade do que de outras categorias profissionais da saúde, pelo fato de ter assumido o controle de estoque, manutenção e validade da vacinas, cobertura vacinal, técnicas de manipulação e aplicação, atividades educativas junto à clientela e o treinamento e supervisão do pessoal auxiliar.

É importante destacar que mesmo as enfermeiras que trabalham no Programa Saúde em Casa (PSC) desenvolvem poucas atividades voltadas para à saúde coletiva, pois gastam grande parte do seu tempo realizando consultas de enfermagem a pacientes com quadro clínico de pequena e moderada complexidade. Neste Programa também a consulta de enfermagem tem finalidade de auxiliar o médico no atendimento da grande demanda espontânea.

Em sintese, os dados mostram que as enfermeiras dedicam grande parte do seu tempo em atividades de organização da infra-estrutura, planejamento do serviço, e planejamento do trabalho da equipe de enfermagem. O desenvolvimento cotidiano dessas atividades confirmam a sua função de coordenação de todo o serviço, e de articulação, principalmente do trabalho médico com o da enfermagem. Fica evidente também que as enfermeiras desenvolvem poucas atividades especificas, sendo a consulta de enfermagem a atividade mais valorizada, e as voltadas para a saúde coletiva.

\section{GRÁFICO 2 -FREQÜÊNCIA DAS ATIVIDADES RARAMENTEDESENVOLVIDAS PELAS EN- FERMEIRAS NO COTIDIANO DOS SERVIÇOS DE SAÚDE COLETIVA}

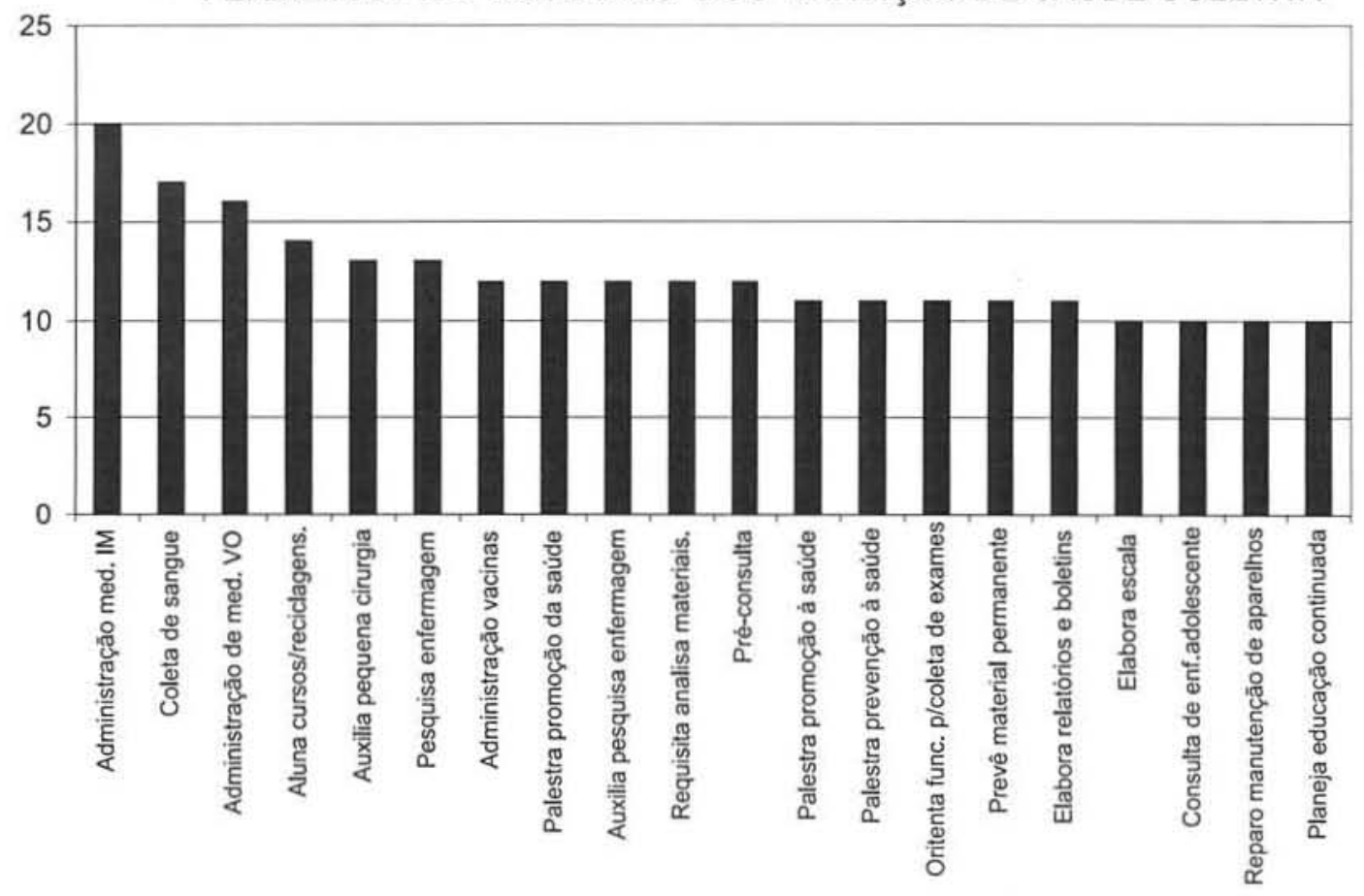


A figura 2 mostra as atividades raramente desenvolvidas pelas enfermeiras. Das atividades voltadas para organização da infra-estrutura e planejamento do serviço identificam-se: a requisição e análise de materiais de consumo, previsão de materiais permanente, reparos e manutenção de aparelhos, elaboração de escalas, relatórios e boletins e orientação de funcionários para coleta de exames.

Das atividades assistenciais complementar ao trabalho do médico raramente desenvolvidas pelas enfermeiras destacam-se: administração de medicamento intramuscular, coleta de sangue, administração de medicamentos via oral e pré-consulta.

Das atividades de caráter educativo e voltadas para à saúde coletiva: palestra de prevenção de doenças e de promoção à saúde. Percebeu-se, nos grupos focais, que as enfermeiras valorizam essas atividades, principalmente as preconizadas pelos Programas instituidos pelo Ministério da Saúde, porém, na prática, pouco desenvolvidas.

Por último, as atividades de capacitação para o desenvolvimento do trabalho: participação em cursos de atualização e reciclagens, realizaçăo e auxilio de pesquisas em enfermagem, auxilio em pesquisas médicas e planejamento de atividades de educação continuada.

Nos grupos focais as enfermeiras expressaram grande insatisfação devido a falta de oportunidade para realizarem cursos de atualização profissional. Para elas, isso demonstra a falta de valorização da profissional pelas instituições de saúde.

Em resumo, constata-se que as enfermeira deixam de realizar atividades extremamente importantes no cotidiano de trabalho, principalmente àquelas mais voltadas para a saúde coletiva. Nesse sentido, faz-se necessário destacar que as enfermeiras, embora assumam diversas atividades administrativas, raramente ou nunca participam das reuniōes da Comissão Local de Saúde.

Além disso, verifica-se que, no cotidiano das unidades de saúde, não estão incluidas as atividades de capacitação para o desenvolvimento do trabalho, o que certamente contribui para a ineficiência do serviço.

\section{GRÁFICO 3-FREQÜÊNCIA DAS ATIVIDADES DIARIAMENTE DESENVOLVIDAS PELOS AUXILIARES DE ENFERMAGEM NO COTIDIANO DOS SERVIÇOS DE SAÚ- DE COLETIVA}

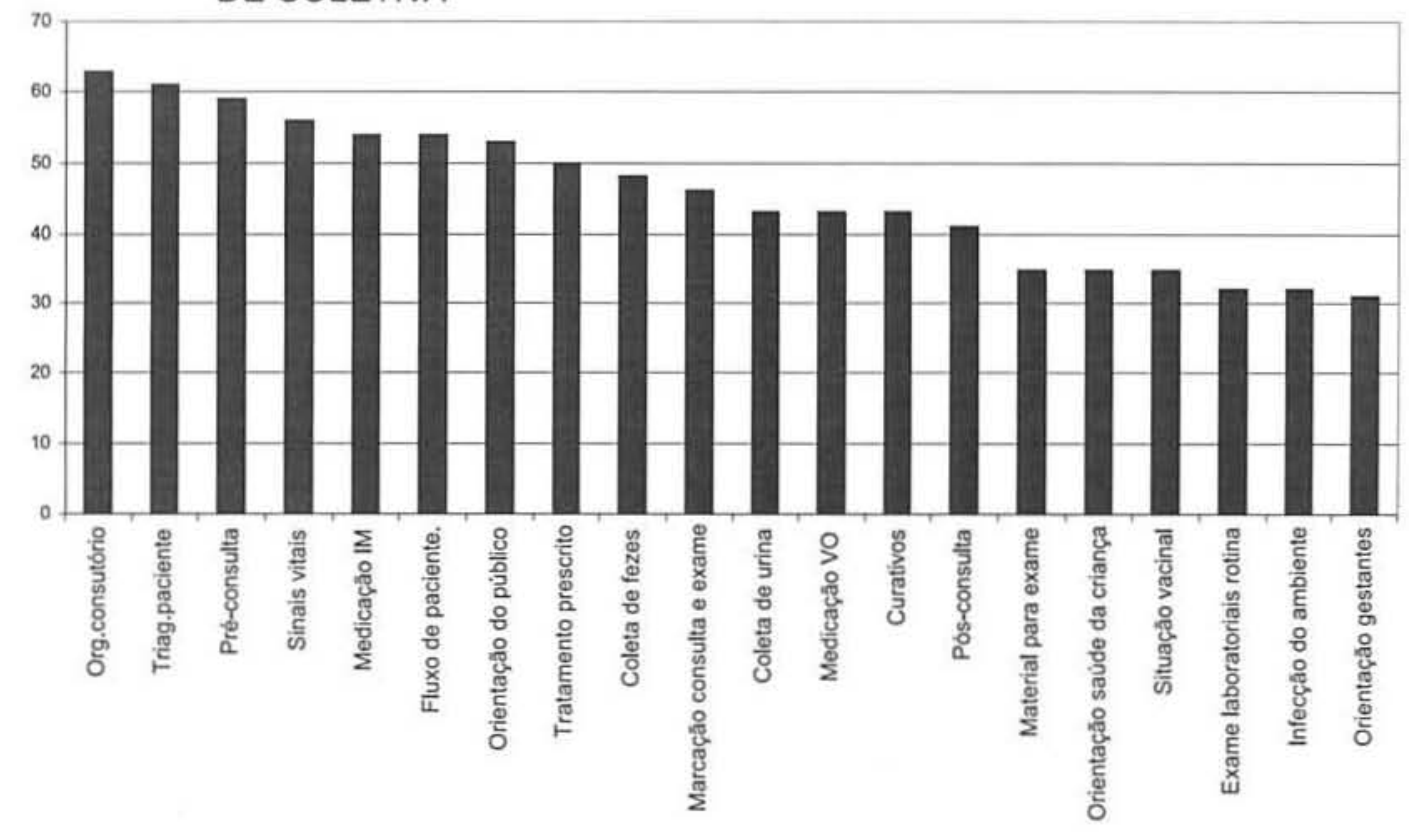


No gráfico 3 identificam-se as atividades diariamente desenvolvidas pelos auxiliares de enfermagem. Das atividades de organização da infra-estrutura e planejamento do serviço destacam-se: organização de consultório, triagem de pacientes, controle do fluxo de pacientes, orientação do público, marcação de consulta e exame, controle de infecção do ambiente.

Das atividades assistenciais de caráter complementar ao trabalho do médico: préconsulta, verificação de sinais vitais, administração de medicamentos intramuscular, administração de tratamento prescrito, coleta de fezes e urina, administração de medicação via oral, curativos, pós consulta, coleta de material para exames e exames laboratoriais de rotina. Das atividades de caráter educativo e atividades voltadas para à saúde coletiva: avaliação da situação vacinal, orientação da saúde da criança e orientação à gestante.

Analisando-se as atividades desenvolvidas pelos auxiliares de enfermagem, verifica-se que esses trabalhadores são os que mais contribuem para que o atendimento médico se processe, pois assumem a organização de toda a infra-estrutura necessária e ainda executam grande número de atividades assistenciais complementares.

Nas sessões de grupos focais, os auxiliares de enfermagem destacaram que grande parte das atividades desenvolvidas nos serviços de saúde baseiam-se nos Programas elaborados pelo Ministério da Saúde e algumas pela Secretaria da Saúde do DF.

\section{GRÁFICO 4-FREQÜÊNCIADAS ATIVIDADES RARAMENTE DESENVOLVIDAS PELOS AUXILI- ARES DE ENFERMAGEM NO COTIDIANO DOS SERVIÇOS DE SAÚDE COLE- TIVA}

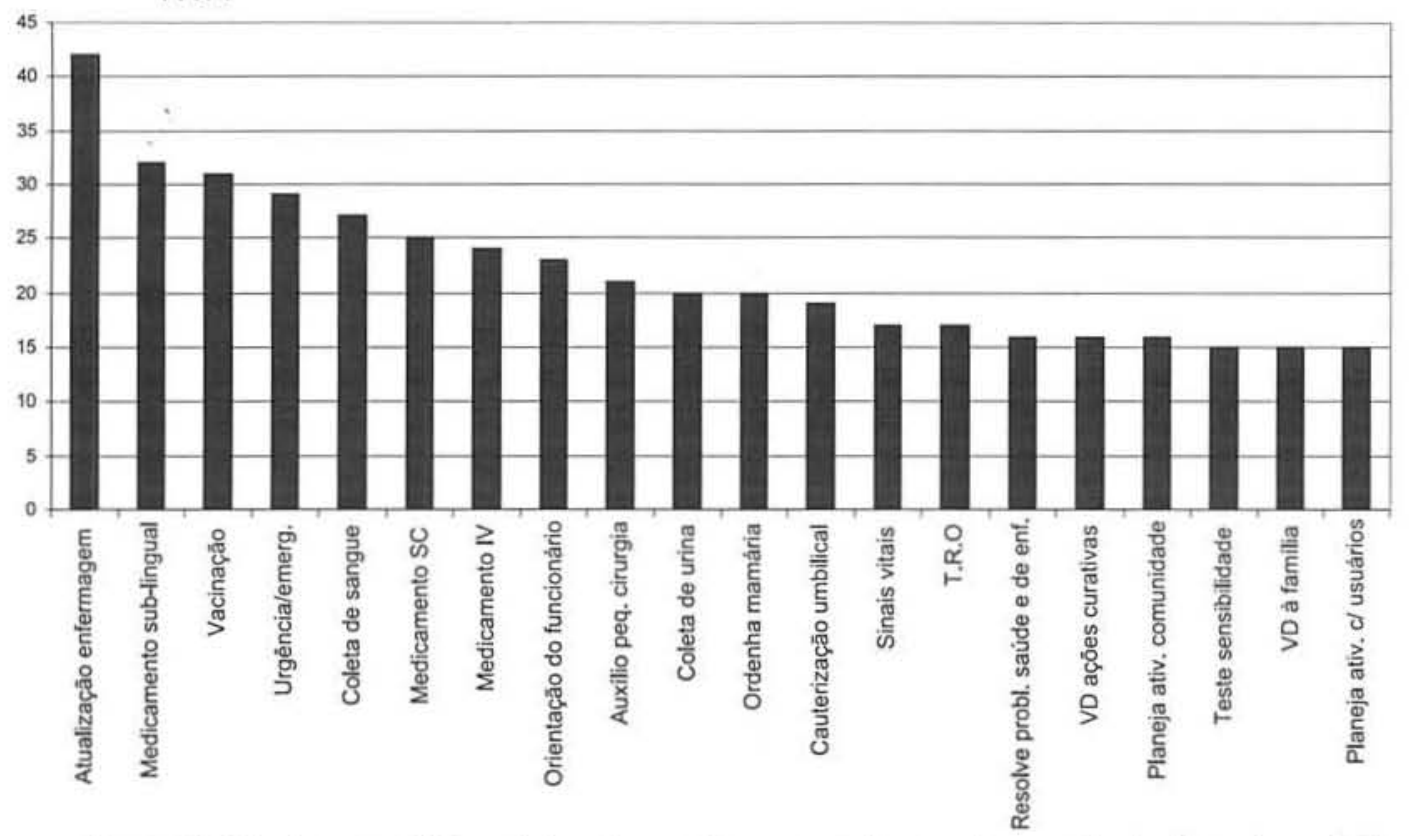

Das atividades assistenciais de caráter complementar ao trabalho do médico identificou-se que os auxiliares raramente realizam destacam-se: administração de medicamentos sublingual, administração de medicamento subcutânea e intravenosa, coleta de sangue, auxilio a pequena cirurgia, coleta de urina, ordenha mamária, cateterização umbilical, teste de sensibilidade. Uma possivel explicação para a baixa freqüência destes procedimentos seria o fato de serem pouco realizados nos Centros e Postos de saúde.

Os auxiliares raramente executam os procedimentos de maior complexidade e que são assumidos pelas enfermeiras, a saber: atendimento de urgência/emergência, TRO e identificação, análise e propostas de soluçöes para os problemas de saúde e de enfermagem. 
Por fim, identificou-se como atividades raramente desenvolvidas pelo auxiliar de enfermagem a vacinação e a verificação de sinais vitais. A primeira porque geralmente existe, nos centros $e$ postos de saúde, um funcionário fixo, ou seja, aquele que recebeu treinamento especifico para tal; a segunda, por ser desenvolvida pelos agentes de saúde.

Das atividades de caráter educativo e atividades voltadas para à saúde coletiva os auxiliares raramente realizam as seguintes atividades: visita domiciliar para realizar ações curativas, planejamento de atividades junto à comunidade, visita domiciliar à familia e planejamento de atividades junto aos usuários.

Fica evidente que os auxiliares de enfermagem quase não desenvolvem atividades fora das unidades de saúde; consequentemente, são os que menos conhecem a realidade vivenciada pela comunidade de que cuidam.

\section{GRÁFICO 5 - FREQÜÊNCIA DAS ATIVIDADES DIARIAMENTE DESENVOLVIDAS PELOS AGENTES DE SAÚDE NO COTIDIANO DOS SERVIÇOS DE SAÚDE COLETIVA}

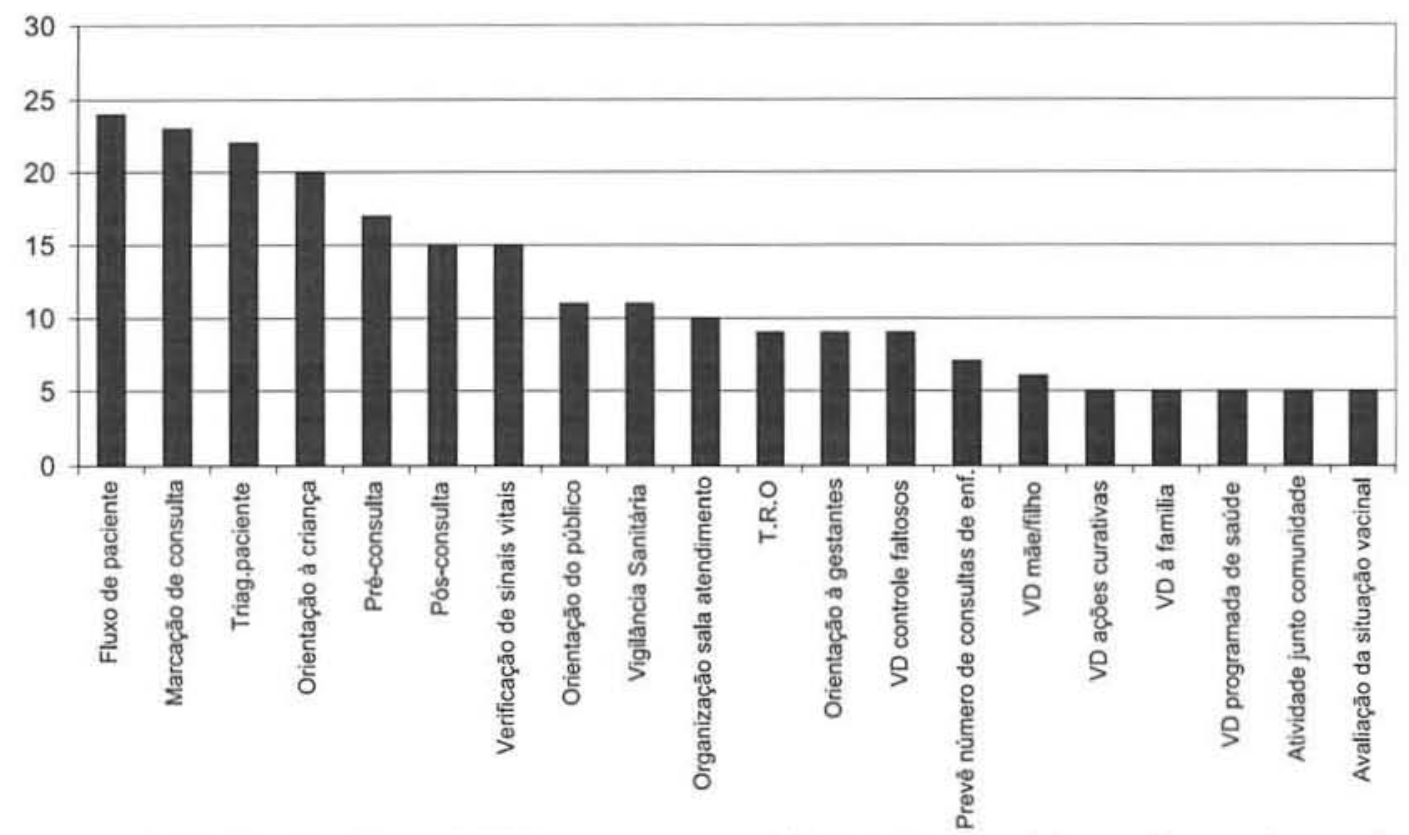

Inicialmente, faz-se necessário esclarecer que houve um aumento no número de agentes de saúde no DF com a criação, em 1997, do Programa Saúde em Casa (PSC). De acordo com o programa, esses trabalhadores deveriam ser membros da comunidade, pois esse requisito ajudaria na identificação de suas necessidades de saúde, mas não precisariam ter formação especifica na área de enfermagem (SANTOS; SHIMIZU, 1999).

$\mathrm{Na}$ figura 5 pode-se visualizar as atividades diariamente desenvolvida pelos agentes de saúde. Das atividades de organização da infra-estrutura e planejamento do serviço destacamse: controle do fluxo de paciente, marcação de consulta, triagem de paciente, orientação do público, organização da sala de atendimento e previsão do número de consultas de enfermagem.

Das atividades assistenciais de caráter complementar ao trabalho do médico destacam-se: pré-consulta, pós- consulta, verificação de sinais vitais e Terapia de Reidratação Oral (TRO).

Das atividades de caráter educativo e atividades voltadas para à saúde coletiva destacam-se: orientação à criança, orientação à gestante, vigilância sanitária, visita domiciliária para controle de faltosos, visita domiciliária à familia, visita domiciliária programada de saúde, 
atividade junto a comunidade e avaliação da situação vacinal.

Analisando-se as atividades desenvolvidas pelos agentes de saúde, verifica-se que auxiliam naquelas voltadas para a organização e atendimento da demanda espontânea do serviço.

Além disso, fica evidente que são eles os que mais desenvolvem atividades de atendimento aos pacientes fora das unidades de saúde. Destaca-se que são os agentes de saúde do PSC os que mais freqüentemente realizam essas atividades. Durante a sessão de grupo focal, esses trabalhadores expressaram grande satisfação em cuidar da comunidade onde residem.

\section{GRÁFICO 6-FREQÜÊNCIA DAS ATIVIDADES RARAMENTE DESENVOLVIDAS PELOS AGENTES DE SAÚDE NO COTIDIANO DOS SERVIÇOS DE SAÚDE COLETIVA}

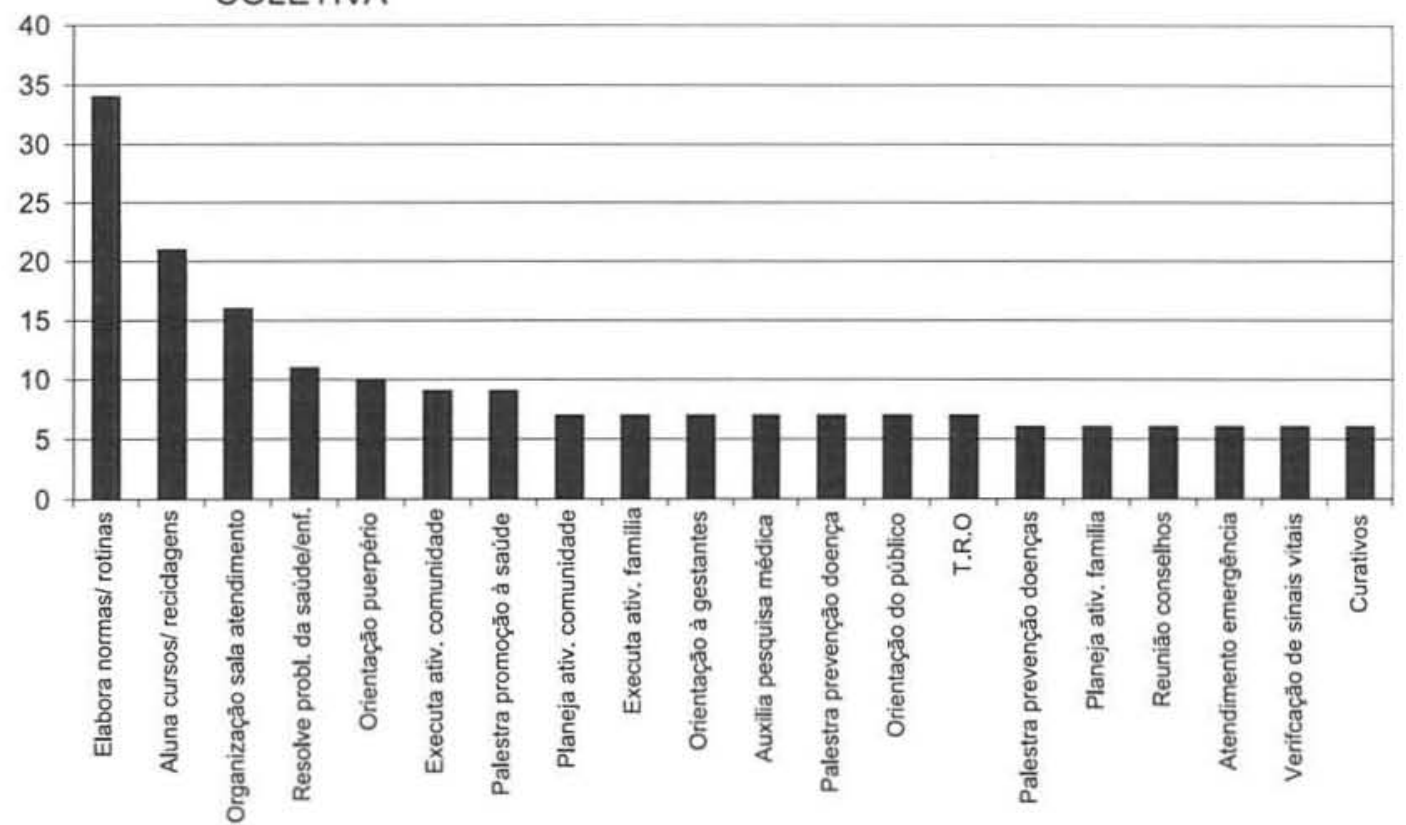

Na figura 6 estão demonstradas as atividades raramente desenvolvidas pelos agentes de saúde. Das atividades de organização da infra-estrutura e planejamento do serviço destacam-se: elaboração de normas e rotinas, organização da sala de atendimento, orientação do público, atendimento de urgência e emergência.

Das atividades assistenciais de caráter complementar ao trabalho do médico destacam-se: Terapia de Reidratação Oral (TRO), verificação de sinais vitais, curativos, resolução de problemas de saúde e de enfermagem.

Das atividades de caráter educativo e atividades voltadas para à saúde coletiva destacam-se: orientação às puérperas, palestra de promoção à saúde, planejamento e execução de atividades junto à comunidade, execução de atividades junto à comunidade, orientação de atividades às gestantes, palestra de prevenção de doenças, planejamento e execução de atividades com as familias e, por fim, participação em reuniōes de Conselhos de Saúde.

Das atividades de capacitação para o desenvolvimento do trabalho: participação em cursos de atualização e reciclagem e auxilio em pesquisas médicas.

Esses dados evidenciam que os agentes de saúde deixam de realizar as atividades de maior complexidade técnica, que são assumidas pelos outros elementos da equipe de enfermagem. Constata-se também que, apesar de serem esses trabalhadores os que mais desenvolvem atividades junto aos usuários, eles deixam de realizar atividades importantes, principalmente a participação no planejamento e execução de atividades de caráter coletivo. 
É interessante notar que os agentes de saúde do PSC são membros da comunidades onde residem, porém não têm tido oportunidade de participar das reuniões da Comissão Local de Saúde.

Para finalizar, faz-se necessário destacar que os agentes de saúde também têm poucas oportunidades para realizar aprimoramento do desempenho profissional e desenvolver atividades que possam contribuir para o desenvolvimento do serviço.

\title{
CONSIDERAÇÕES FINAIS
}

Ao se identificar e analisar as atividades desenvolvidas pelas enfermeiras, auxiliares de enfermagem e agentes de saúde, fica evidente que as práticas de enfermagem estão voltadas principalmente para a organização da infra-estrutura, planejamento do serviço e desenvolvimento de atividades assistenciais de caráter complementar ao trabalho do médico, no sentido de viabilizar o pronto-atendimento médico. É necessário acrescentar que todos os elementos da equipe de enfermagem apontaram que grande parte dessas atividades visam atender a grande demanda espontânea presente nos serviços de saúde. Nesse sentido, é importante destacar que essas atividades espelham o modelo assistencial vigente na Regional Norte do Distrito Federal, que se assenta em uma prática de atenção curativa, individualizada e focal.

Identificou-se também que as poucas atividades que se caracterizam como atividades mais voltadas para a saúde coletiva, como visita domiciliária, palestras de prevenção e promoção da saúde, não cumprem esta finalidade, pois, na maioria das vezes, são realizadas isoladamente e para atender somente às necessidades mais imediatas. Há que se destacar que grande parte dessas atividades são desenvolvidas pelos agentes de saúde.

Foi possivel perceber que as enfermeiras deixam de realizar atividades extremamente importantes no cotidiano de trabalho, principalmente aquelas mais voltadas para a saúde coletiva. Seguindo esta linha de pensamento, faz-se necessário salientar que as enfermeiras precisariam participar de atividades mais estratégicas, como as reuniões da Comissão Local de Saúde e dos Conselhos de Saúde.

Além disso, verifica-se a necessidade de as enfermeiras incluirem no cotidiano das unidades de saúde as atividades para capacitação profissional e desenvolvimento do trabalho, envolvendo todos os elementos da equipe de enfermagem.

Por fim, conclui-se que, para alcance do modelo sanitário que toma como objeto o fenômeno coletivo do processo saúde-doença, há um longo caminho a ser percorrido e transformado pelas enfermeiras e demais trabalhadores da enfermagem e da saúde.

\begin{abstract}
The purpose of this study is to identify and analyze the activities developed by the nursing team at the Northern Regional Health Unit of the Federal District. A questionnaire, which described a variety of daily nursing activities, was administered to 29 nurses, 91 nurse assistants and 42 health care agents. Focus group sessions for each of the professional categories were also conducted. Results showed that all members of the nursing team concentrate their effort in organizing, controling and attending spontaneous demands as well as providing support to doctors. As a consequence, these professionals prioritize individual attendance to detriment of collective practice.
\end{abstract}

KEYWORDS: nursing practice, Collective Health, nursing

RESUMEN: Este estudio identifica y analiza las acciones desarrolladas por equipo de enfermeria en salud colectiva de la Regional Norte de Salud del Distrito Federal. La recolección de datos se realizó mediante cuestionario con la descripción de las diversas actividades desarrolladas diariamente por el equipo de enfermería, que totaliza 29 enfermeras, 91 auxiliares de enfermeras y 42 agentes de salud. Se organizaron grupos de acuerdo a las categorias profesionales. Los resultados demostraron que todos los miembros del equipo de enfermeria desarrollan con más frecuencia tareas con la 
finalidad de ayudar las actividades médicas. Se concluye que el equipo realiza grandes esfuerzos en el trabajo diario de las unidades de salud para organizar, atender y controlar la gran demanda expontánea, y en consecuencia, tiene que priorizar la atención individual en detrimento de lo colectivo.

PALABRAS CLAVE: actividades de enfermeria, salud colectiva, enfermeria

\section{REFERÊNCIAS BIBLIOGRÁFICAS}

ALMEIDA et al. O trabalho de enfermagem e a sua articulação com o processo de trabalho em saúde coletiva: rede básica de saúde. In: ALMEIDA, M. C. P. de; ROCHA, S. M. O trabalho da enfermagem. São Paulo: Cortez, 1997. p. 61-112.

ARAUJO, M. J. S. A consulta de enfermagem no contexto da prática de enfermagem. Brasilia: ABEn, 1991. p. 30-45. Série documental: I.

ANTUNES, M. J. M. ; SILVA, I. A.; EGRY, E. Y. ; SENA, R. R. de; ALMEIDA, M. C. P. de. Manual do Pesquisador, orientaçăo para trabalho de campo. Brasilia: ABEn, 1997. 93p.

BERTOLOZZI et al. A classificação das práticas de enfermagem em saúde coletiva: cenário São Paulo . In: ANTUNES, M. J. ; CHIANCA, T. C. M. A classificação internacional das práticas de enfermagem em saúde coletiva: CIPESC. Brasilia: ABEn, 1999 p. 245-269. (Série didática: Enfermagem no SUS).

FERREIRA, S. L. A mulher e os serviços públicos de saúde. In: ALMEIDA, M. C. P. de; ROCHA, S. M. O trabalho da enfermagem. São Paulo: Cortez, 1997. p.175-227.

MISHIMA et al. A classificação internacional para a prática de enfermagem em saúde coletiva no Brasil- CIPESC - apresentando o cenário de pesquisa do municipio de Ribeirão Preto - São Paulo. In: ANTUNES, M. J. ; CHIANCA, T. C. M. A classificaçäo internacional das práticas de enfermagem em saúde coletiva: CIPESC. Brasilia: ABEn. 1999. p. 204-244. (Série didática: Enfermagem no SUS).

SEMINÁRIO NACIONAL SOBRE CURRICULO DO CURSO DE GRADUAÇÃO EM ENFERMAGEM. 1986. Escola de Enfermagem da Universidade de São Paulo, São Paulo.

SILVA, E. R. da ; SHIMIZU, H. E. Breve histórico da assistência à saúde no Distrito Federal e caracterização de cenário na perspectiva da saúde coletiva. In: A classificação internacional das práticas de enfermagem em saúde coletiva: CIPESC. CHIANCA, T. C. M.; ANTUNES, M. J. M.(Org) Brasilia: ABEn, 1999. p.66-79. (Série Didática: Enfermagem no SUS).

SHIMIZU, H. E. ; SILVA, E. R. A percepção de gerentes e gestores sobre a assistência à saúde coletiva no Distrito Federal. Trabalho apresentado no 10. Seminário Nacional de Pesquisa em Enfermagem, Porto Alegre, 1999. 10 p. Mimeogr.

Recebido em setembro de 2001

Aprovado em dezembro de 2001 